\title{
Multiscale three-point velocity increment correlation in turbulent flows
}

\author{
Siyuan Yao ${ }^{\mathrm{a}}$, Le Fang ${ }^{\mathrm{a}, \mathrm{b}, *}$, Jinming $\mathrm{Lv}^{\mathrm{a}}$, Jianzhao $\mathrm{Wu}^{\mathrm{a}}$, Lipeng $\mathrm{Lu}^{\mathrm{c}}$ \\ a Laboratory of Mathematics and Physics, Ecole Centrale de Pékin, Beijing University of Aeronautics and Astronautics, Beijing 100191, China \\ b The State Key Laboratory of Nonlinear Mechanics, Institute of Mechanics, Chinese Academy of Sciences, Beijing 100190, China \\ c School of Energy and Power Engineering, Beijing University of Aeronautics and Astronautics, Beijing 100191, China
}

\section{A R T I C L E I N F O}

\section{Article history:}

Received 31 August 2013

Accepted 13 January 2014

Available online 22 January 2014

Communicated by F. Porcelli

\section{Keywords:}

Three-point velocity correlation

Turbulence

Large Eddy Simulation

Velocity increment

Multiscale analysis

\begin{abstract}
A B S T R A C T
The three-point velocity increment correlation function is proposed to represent the multiscale correlations in turbulent flows. The inertial-inertial correlation and the inertial-dissipative correlation are discussed due to their endogenetic properties in turbulence and their roles in large-eddy simulation. The zero-correlation points are then emphasized as equilibrium points between them. The credibility of this theoretical result is numerically verified in both isotropic and anisotropic flows. Results imply the universality of this zero-correlation scaling in different turbulent flows. This work is expected to be a dependable theoretical base for creating multiscale subgrid models in large-eddy simulation.
\end{abstract}

(c) 2014 Elsevier B.V. All rights reserved.

\section{Introduction}

The Large Eddy Simulation (LES) technique has been developed in the last 50 years, yet till now it may not be regarded as a reliable tool in practical computations for engineering problems [1]. The remarks, from the engineering community, are mostly on the unclear criterions of choosing a proper subgrid model in a typical problem: on the physical level, this reality should be due to the fact that the subgrid models, which should represent the multiscale energy transfer relations, are usually not universal in complex flows.

In general, the process of formulating a subgrid model can be divided into two steps [2]: (i) to assume a similarity between the resolved and subgrid scales; (ii) to use a certain physical law to calculate the ratio of this similarity, or say to make a closure. The important thing is that this physical law should be universal in reality. For example, if this law behaves differently in isotropic and anisotropic turbulent flows, it would not be the best choice [3-5].

There are two physical laws which are widely used in current subgrid models: one is the $-5 / 3$ slope of energy spectrum [6]; the other one is the (traditional or anomalous) scaling law of the twopoint second-order structure functions [6-8]. The spirit of them are similar on claiming a quantitative multiscale relation. Compar-

\footnotetext{
* Corresponding author at: Laboratory of Mathematics and Physics, Ecole Centrale de Pékin, Beijing University of Aeronautics and Astronautics, Beijing 100191, China. Tel.: +861082316629.

E-mail address: le.fang@buaa.edu.cn (L. Fang).
}

ing to the former one in spectral space, the latter is a relation in physical space and is then more appropriate for practical applications. However, this scaling law is not universal. Many researchers have shown that, although the small scale fluctuations always tend to be isotropic, the two-point scaling law is definitively affected by the large-scale anisotropy [9-12]. Therefore, effects of finding a more universal multiscale relation will be helpful.

The recent work of Marusic et al. [13] shed light on the multiscale similarity in wall-bounded turbulence, by considering the correlation between large- and small-scale motions. However, this work did not distinguish the wall effect from the more common multiscale correlation, i.e., the endogenetic correlation in homogeneous isotropic turbulence (HIT). It should be clarified which types of multiscale correlations always exist in turbulent flows and are not consequences of the wall effect. Therefore, we would like to investigate this endogenetic correlation by revisiting the three-point velocity increment correlation, which was firstly defined by L'vov et al. [14] and analyzed by Benzi et al. [15]. Our work expands the results of Benzi et al. from one dimension to three dimensions, and also shows their universality in anisotropic turbulence by processing the Direct Numerical Simulation (DNS) results. We then conclude that the aim of the present work is a theoretical approach to investigate the multiscale correlation, focusing on finding the universal physical behavior of turbulent flows.

\section{Three-point velocity increment correlation function}

In the following, we will revisit the work of Benzi et al. for the one-dimensional three-point velocity increment correlation 
function, and discuss its behaviors in different scales. Then a threedimensional formulation is derived by expanding this work.

Following Benzi et al., the one-dimensional three-point velocity correlation function can be defined as (see Fig. 1 as a sketch)

$D_{A B \leftrightarrow B C}(r, l)=\left\langle\left(u_{A}-u_{B}\right)\left(u_{B}-u_{C}\right)\right\rangle$,

where $r$ and $l$ define the two distances $A B$ and $B C$ respectively. $A$, $B$ and $C$ are located at one straight line in HIT. The corresponding velocity components along this line are denoted as $u_{A}, u_{B}$ and $u_{C}$ respectively. In the following $r$ and $l$ are assumed to be normalized by the Kolmogorov scale $\eta$. Physically we can then consider this definition as the correlation between the velocity increments of different scales $r$ and $l$. Although the definition of this correlation is distinct from the signal processing method of Marusic et al., we could say that the main ideas are similar. In HIT, traditional theories of two-point second-order structure function lead to

$\left\langle\left(u_{A}-u_{B}\right)^{2}\right\rangle=D_{l l}(r), \quad\left\langle\left(u_{B}-u_{C}\right)^{2}\right\rangle=D_{l l}(l)$,

$\left\langle\left(u_{A}-u_{C}\right)^{2}\right\rangle=D_{l l}(r+l)$,

with \langle\rangle the ensemble average, and $D_{l l}()$ the second-order longitudinal structure function. From Eqs. (1) and (2) we can simply obtain

$D_{A B \leftrightarrow B C}(r, l)=\frac{1}{2}\left(D_{l l}(r+l)-D_{l l}(r)-D_{l l}(l)\right)$.

This formula deduces the three-point correlation from the traditional two-point structure functions and then allows the following theoretical analysis. In addition, this formula shows a scaling behavior among three different scales, i.e., $r, l$, and $r+l$, thus a

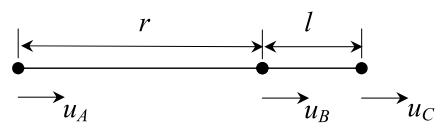

Fig. 1. Sketch of one-dimensional three-point correlation.

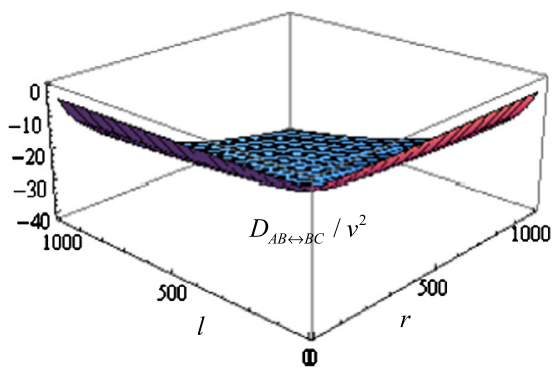

(a) classical scaling law of structure functions is implied here as a multiscale relation.

In the following we will examine this formula by considering the correlation between different scales.

\subsection{Inertial-inertial range correlation}

Assuming extremely high Reynolds number and extremely wide inertial range, we examine the case when all scales $r, l$ and $r+l$ are located in the inertial range, where $D_{l l}(x)=2 v^{2} x^{2 / 3}$ with $v$ the turbulence fluctuation. From Eq. (3) we have

$D_{A B \leftrightarrow B C}(r, l)=v^{2}\left((1+l / r)^{2 / 3}-1-(l / r)^{2 / 3}\right) r^{2 / 3}$.

Fig. 2 shows that the function is negative and monotonically decreasing by either $r$ or $l$, indicating the velocity increments between $u_{A}-u_{B}$ and $u_{B}-u_{C}$ tend to have the opposite signs. Indeed, this fact can be explained that at large scales the three velocities should be approximately zero-average. The scaling of $l / r \rightarrow 0$ was discussed in Ref. [15], and in this letter it will not be focused on.

\subsection{Inertial-dissipative range correlation}

The second case assumes that $r$ and $r+l$ are located in the inertial range, and $l$ in the dissipative range, say $l \ll 1$ and $r \gg 1$. This leads to

$D_{l l}(r)=2 v^{2} r^{2 / 3}, \quad D_{l l}(r+l)=2 v^{2}(r+l)^{2 / 3}$,

$D_{l l}(l)=\frac{1}{15} v^{2} l^{2}$.

From Eq. (3) we have

$D_{A B \leftrightarrow B C}(r, l)=v^{2}\left((1+l / r)^{2 / 3}-1-\frac{1}{30}(l / r)^{2} r^{4 / 3}\right) r^{2 / 3}$,

with the results shown in Fig. 3. In Fig. 3(b) we fix $r=10$ as an example. It is observed that the values in Fig. 3 are all positive,

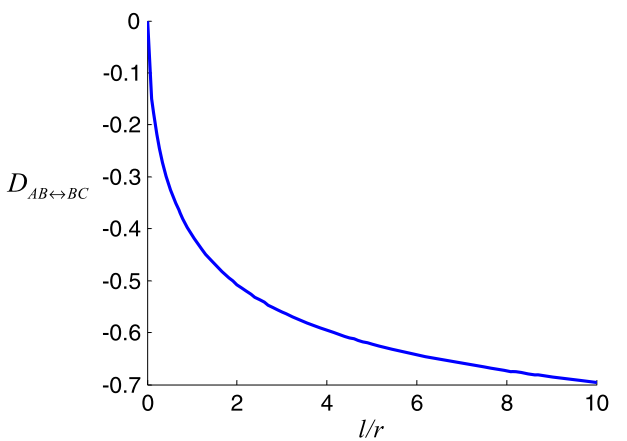

(b)

Fig. 2. One-dimensional inertial-inertial range correlation. (a) Three-dimensional figure $D_{A B \leftrightarrow B C} / v^{2}$ in terms of $r$ and $l$. (b) $D_{A B} \leftrightarrow B C / v^{2}$ in terms of $l / r$, with $r=10$.

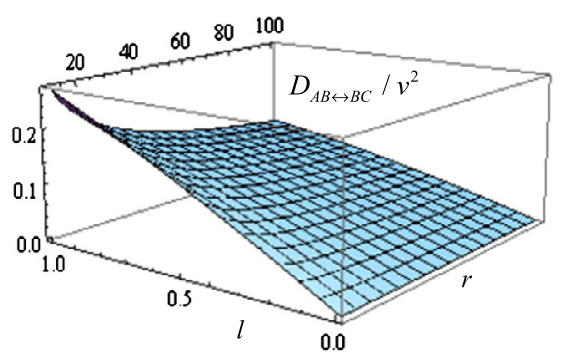

(a)

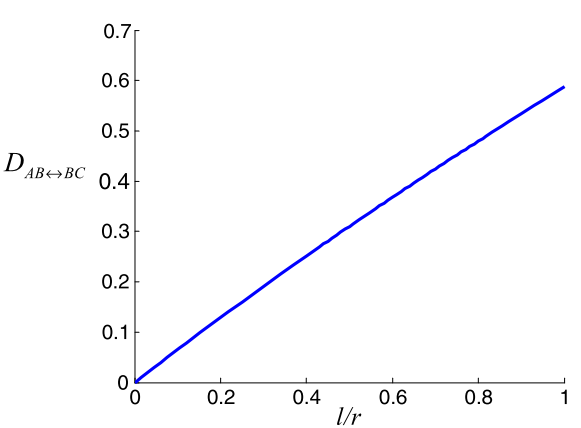

(b)

Fig. 3. One-dimensional inertial-dissipative range correlation. (a) Three-dimensional figure $D_{A B \leftrightarrow B C} / v^{2}$ in terms of $r$ and $l$. (b) $D_{A B \leftrightarrow B C} / v^{2}$ in terms of $l / r$, with $r=10$. 


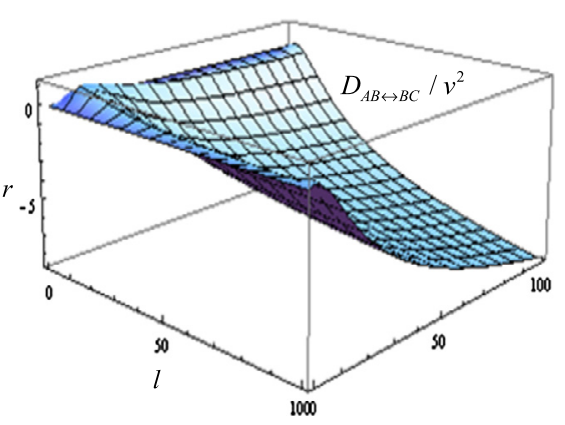

(a)

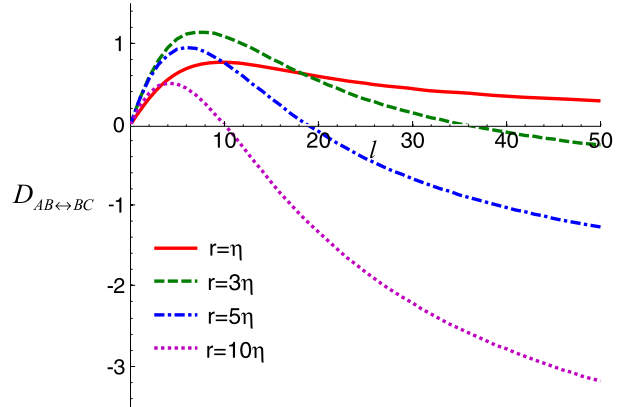

(b)

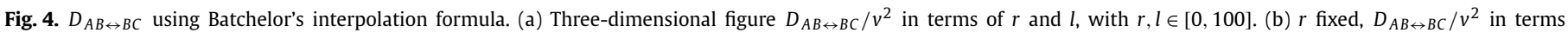
of $l$.

which could be explained that the small-scale (i.e., scale $l$ ) velocity increment tends to follow the large-scale (i.e., scale $r$ ) motion, as a natural consequence of continuous functions. This inertialdissipative correlation was not mentioned in Ref. [15].

Comparing these two typical cases, we conclude that the endogenetic multiscale correlations can be either negative or positive, and reflect different physical significances. Note that we only consider the cases between an inertial range scale and a smaller scale, because this is exactly the spirit of LES that filters the turbulence in inertial range and models the smaller-scale fluctuations.

\subsection{Using Batchelor's interpolation formula}

Besides the two typical cases discussed above, Batchelor's interpolation formula allows the study of the transition between dissipative and inertial scales. This formula reads [16]

$D_{l l}(x)=v^{2} \frac{2 x^{2 / 3}}{\left(1+(C / x)^{2}\right)^{2 / 3}}$,

where $C \approx 13$ is constant. Here we would like to comment that although this formula is empirical, it is currently one of the most accurate analytical relations which integrate the two single range two-point second-order longitudinal structure functions [17-20].

Similarly we can employ Eqs. (3) and (7) to draw the values of three-point function in Fig. 4. We fix $r$ and change $l$ (see Fig. 4(b)) to study the transition from a dissipative-inertial one to an inertial-inertial one, that is, the value changes from positive to negative when $l$ increases, e.g., when $r=\eta$, the velocity increment $u_{A}-u_{B}$ has a positive correlation with the increment $u_{B}-u_{C}$ when its scale is less than $10 \eta$, and a negative correlation otherwise. It is interesting that when $r=10 \eta$ and $l \approx 27 \eta$, we have $D_{A B \leftrightarrow B C}=0$, which indicates a statistical independence between these two scales. Strictly speaking, this independence is a statistical balance between the dissipative-inertial and inertialinertial interactions. This would be important in LES applications, because when we take a filter size in inertial range (corresponding to the scale $r$ here), we should know how to model the smallerscale parts, i.e., which part is positively dependent to the resolved motion and which part negatively dependent. This zero-correlation point will be carefully examined in Section 3 .

\subsection{Three-dimensional three-point velocity increment correlation}

In order to examine the behavior of anisotropic turbulent flows in the numerical tests, a theoretical comparison of the threedimensional correlation function in HIT should be helpful. The onedimensional definition is then expanded to the three-dimensional three-point velocity increment (see Fig. 5):

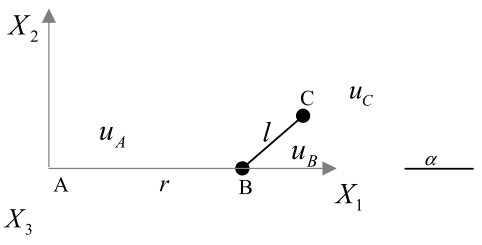

Fig. 5. Sketch of three-dimensional three-point correlation $u_{A}, u_{B}$ and $u_{C}$ are collinear in the direction of vector $\vec{a}$, the angle between $\overrightarrow{A B}$ and $\overrightarrow{B C}$ is $\alpha$.

$$
\begin{aligned}
D_{A B \leftrightarrow B C, a}(r, l, \alpha)= & \left\langle\left(u_{A}-u_{B}\right)\left(u_{B}-u_{C}\right)\right\rangle_{a} \\
= & \left\langle u_{A, i} u_{B, j}\right\rangle \cdot e_{i a} e_{j a}-\left\langle u_{A, i} u_{C, k}\right\rangle \cdot e_{i a} e_{k a} \\
& +\left\langle u_{B, j} u_{C, k}\right\rangle \cdot e_{j b} e_{k a}-v^{2},
\end{aligned}
$$

where the subscript ${ }_{a}$ stands for the velocity direction fixed in the direction $\vec{a}$. The velocity component in the direction $\vec{a}$ of point $A$ can be written as $u_{A, a}=u_{A, i} e_{i a}$ with $u_{A, i}$ the velocity projection of point $A$ on the axis $i, e_{i a}$ the cosine value of the angle between the vector $\vec{a}$ and the axis $i .\left\langle u_{A, i} u_{B, j}\right\rangle,\left\langle u_{B, j} u_{C, k}\right\rangle$ and $\left\langle u_{A, i} u_{C, k}\right\rangle$ can be expressed by the longitudinal and transverse structure functions $D_{l l}$ and $D_{n n}$. Following Ref. [21], we have

$\left\langle u_{A, i} u_{B, j}\right\rangle=v^{2}\left(\frac{r_{i} r_{j}}{r^{2}}(f(r)-g(r))+g(r) \delta_{i j}\right)$,

where $r_{i}$ is the normalized projective length on axis $i$ between $A$ and $B, f(r)$ and $g(r)$ are nondimensional longitudinal and transverse correlation functions respectively. The other two functions $\left\langle u_{B, j} u_{C, k}\right\rangle$ and $\left\langle u_{A, i} u_{C, k}\right\rangle$ can be derived similarly.

In the HIT we can write the transverse structure function as

$D_{n n}(x)=D_{l l}(x)+\frac{x}{2} D_{l l}^{\prime}(x)$.

Since

$f(x)=1-\frac{D_{l l}(x)}{2 v^{2}}$ and $g(x)=1-\frac{D_{n n}(x)}{2 v^{2}}$,

we have

$\left\langle u_{A, i} u_{B, j}\right\rangle=\frac{r_{i} r_{j}}{4 r} D_{l l}^{\prime}+\left(v^{2}-\frac{D_{l l}}{2}-\frac{r}{4} D_{l l}^{\prime}\right) \delta_{i j}$.

As $\left\langle u_{B, i} u_{C, j}\right\rangle$ and $\left\langle u_{A, i} u_{C, j}\right\rangle$ can be deduced similarly, substituting them into Eq. (8), we can finally obtain the expression of $D_{A B \leftrightarrow B C, a}(r, l, \alpha)$. The longitudinal structure function $D_{l l}(x)$ can be replaced with any experimental or empirical formula, such as Batchelor's formula (7).

\subsection{Considering intermittency}

In the previous parts we assume the Kolmogorov 2/3 law in inertial range. Indeed, this may be not appropriate due to the widely 


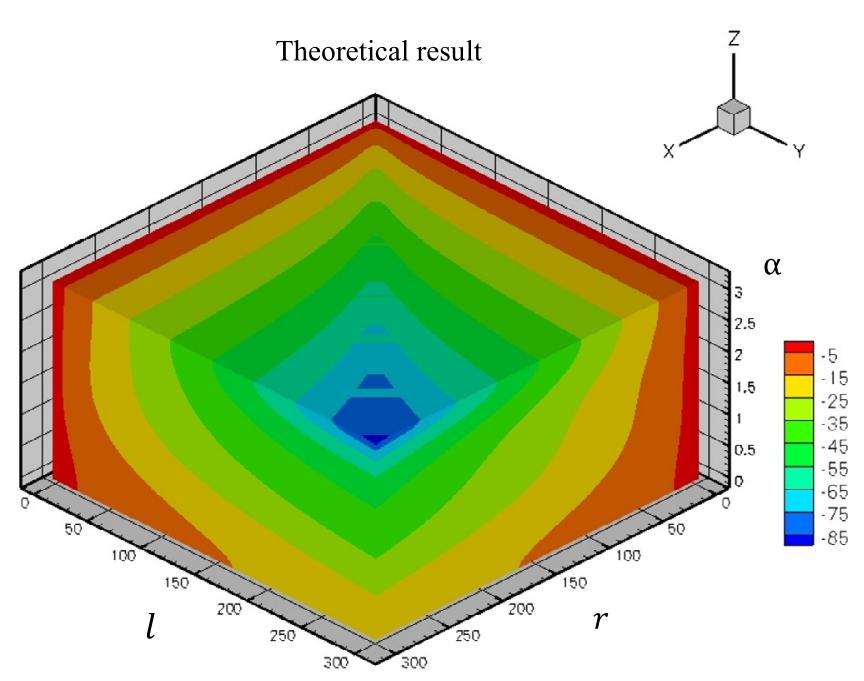

(a)

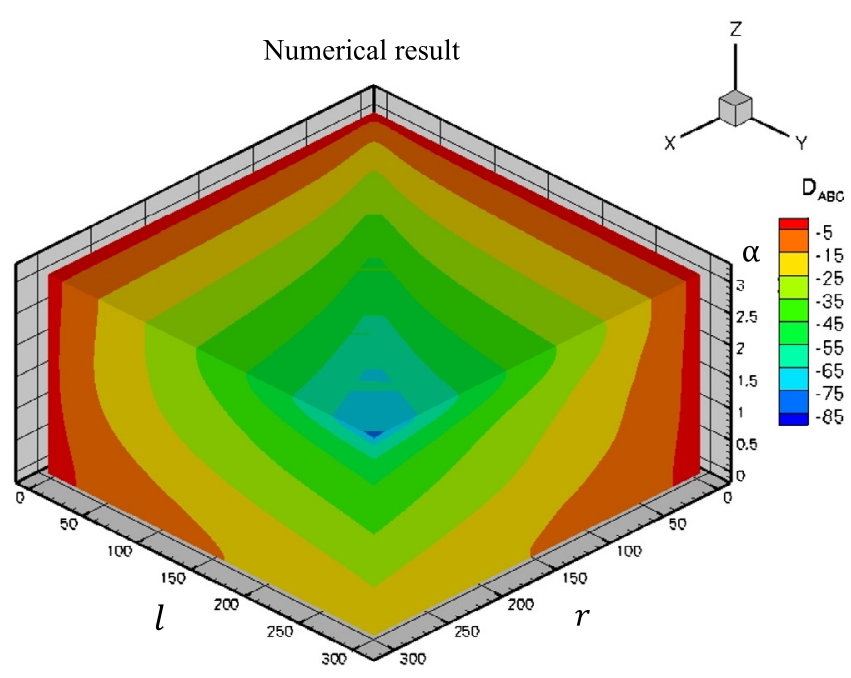

(b)

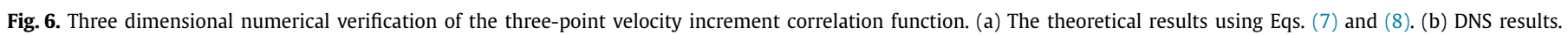

existing intermittency effect $[8,15]$. In case of considering intermittency, another scaling exponent (normally about 0.7 ) instead of the number $2 / 3$ should be employed. It is found that there are no qualitative differences comparing to the above results.

\section{Numerical verification}

Two DNS databases are used in this letter. The first database is the high Reynolds HIT, generated by Gotoh et al. [22]; the other one is a Couette channel flow, as introduced in Ref. [23]. In the HIT case all statistics are made by an average operation in all the three directions. As the theoretical derivation in HIT has already been introduced above, the HIT database is applied to illustrate this theory; by contrast, the channel flow case is employed to investigate the anisotropic behavior.

A general view of the three-point velocity increment correlation function is shown in Fig. 6, focusing on the inertial-inertial correlation when $r$ and $l$ are large. Batchelor's formula is used as a theoretical comparison. Generally the DNS result is quite consistent with the theoretical result, except in the region where $r$ and $l$ are extreme values. This consistency validates both our theory and the DNS results. The slight difference when $r$ and $l$ are large is due to the fact that Batchelor's formula does not consider the finite Reynolds effect at the scales near the energy-containing scale.

A one-dimensional comparison with the theoretical result (Fig. 4(b)) is also presented in Fig. 7 to verify the DNS results. Good agreement between theoretical and numerical results is observed.

As already stated, we are particularly interested in the distribution of the zero-correlation points. From Fig. 8(a) and 8(b), we find the existence of the zero-correlation isosurface, which means the velocity increments are uncorrelated when the parameters are on this surface. The similarity between the theoretical isosurface and the DNS result illustrates the credibility of theoretical derivation again. In the figures, the parameters behind the isosurfaces, i.e., in the same side as the original point, correspond to an inertial-dissipative correlation which leads to positive values of the three-point function; the parameters in the other side contribute an inertial-inertial correlation. Both subfigures show that the zero-correlation points exist when the angle $\alpha$ is smaller than $\pi / 2$. From Fig. 5 this can be understood that in HIT the correlation only exists between both longitudinal directions or both transverse directions. Only in the region $\alpha<\pi / 2$ the three-point tensor (8) can be decomposed to a longitudinal-transverse part and a

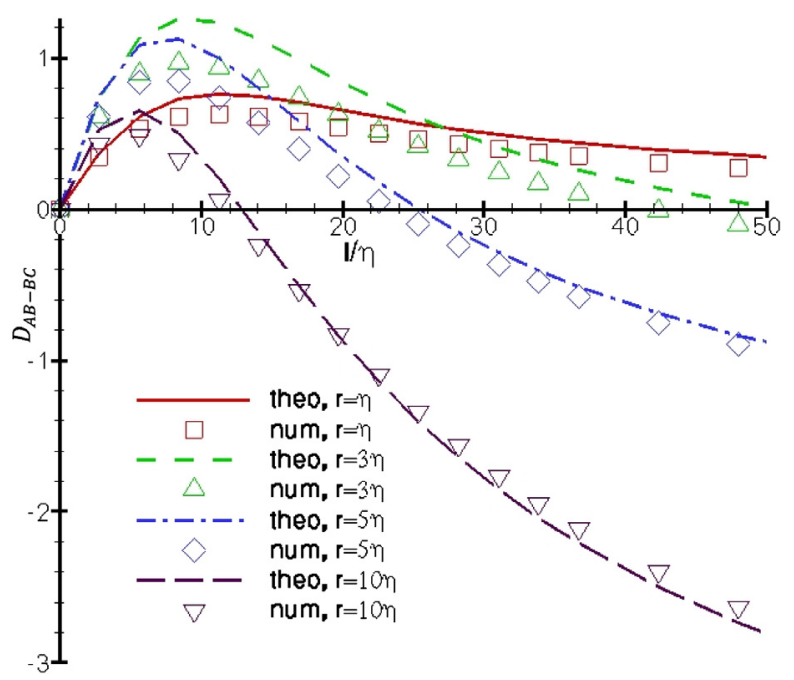

Fig. 7. One-dimensional verification of the three-point velocity increment correlation function. Parameters are the same as in Fig. 4(b).

longitudinal-longitudinal part, where the latter one can contribute an inertial-dissipative correlation. We could then remark that the effect of $\alpha$ can be investigated as a rotation of tensor. Therefore, in the following we only focus at the case when $\alpha=0$, i.e., the one-dimensional case.

In order to examine the different performances of the threepoint correlation function in different types of flow, we observe the zero-correlation line under the condition $\alpha=0$ of DNS data in both HIT and the channel flows, then find the difference by analyzing the scaling of the equipotential line. Similar to the form of scaling law in turbulence, we suppose the zero-equipotential line satisfies $r=l^{n}$, and then define the scaling exponent of a zerocorrelation line as $n=r^{\prime} \cdot l / r=\delta r / \delta l \cdot l / r$.

In the Couette channel flow we only use the central part (i.e., away from walls) of the channel for statistical analysis, where the flow is approximately driven by constant shear. In the following we will denote the streamwise, normal and spanwise directions as $x, y$ and $z$, respectively.

Fig. 9(a) demonstrates the difference between the zero-correlation lines in HIT and in channel turbulent flow. The lines, except in the streamwise direction (i.e., the $x$ direction), are approximately in good agreement, which probably indicates a universal 


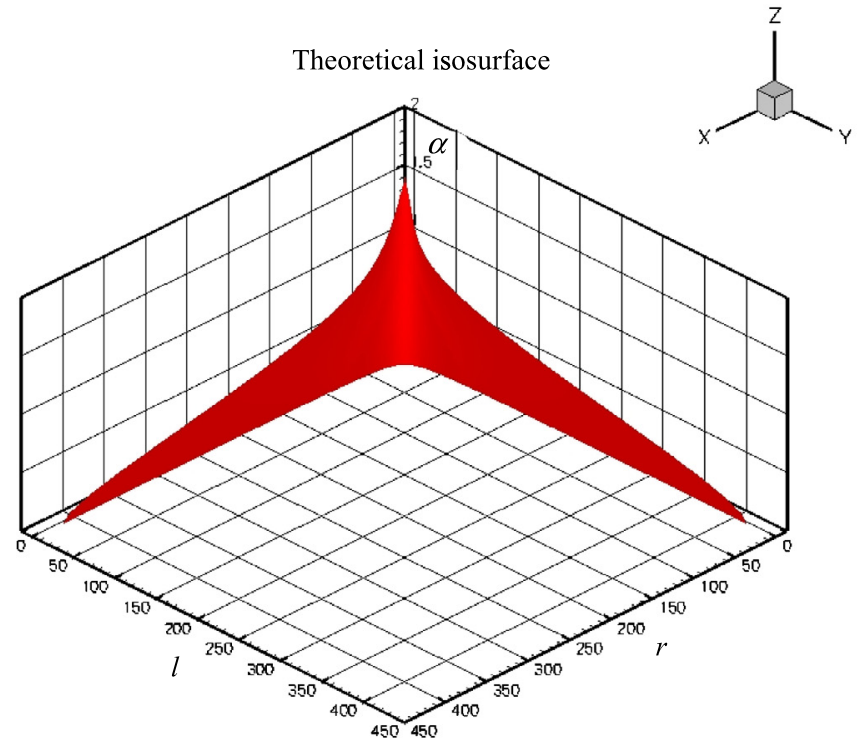

(a)

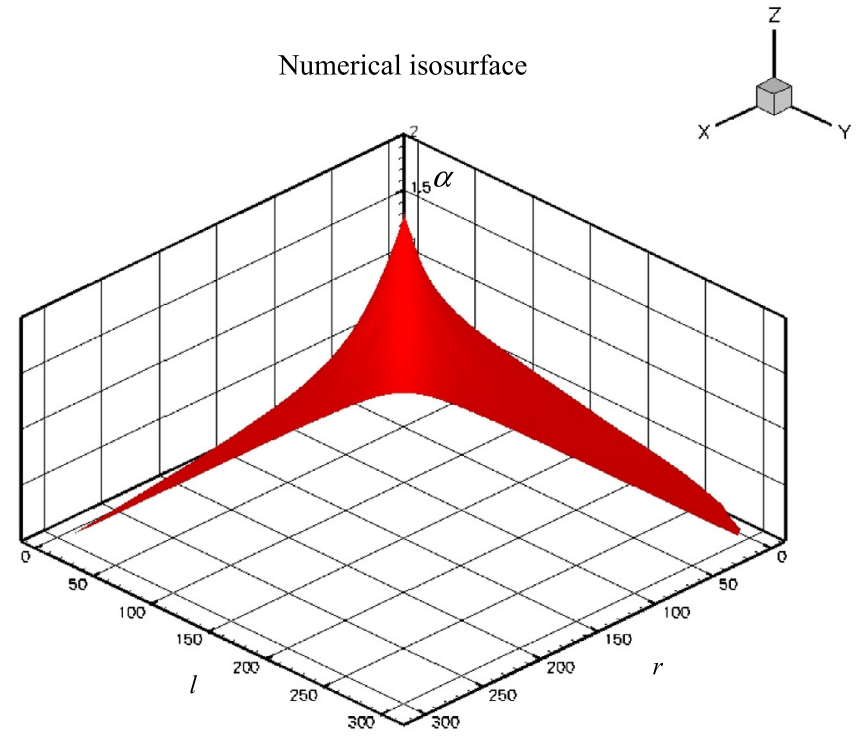

(b)

Fig. 8. Three-dimensional numerical verification of the zero-correlation isosurface. (a) Theoretical results using Eqs. (7) and (8). (b) DNS results.

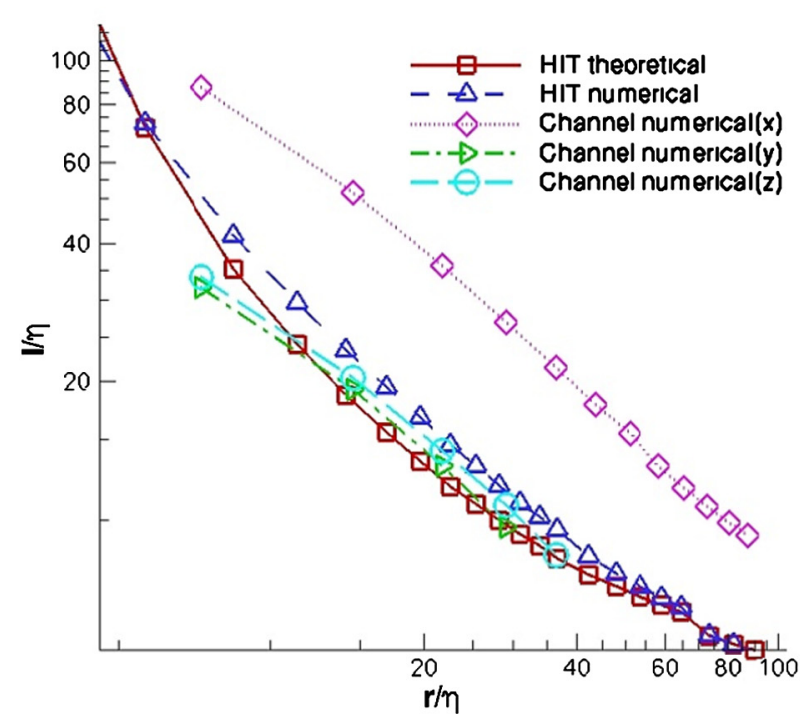

(a)

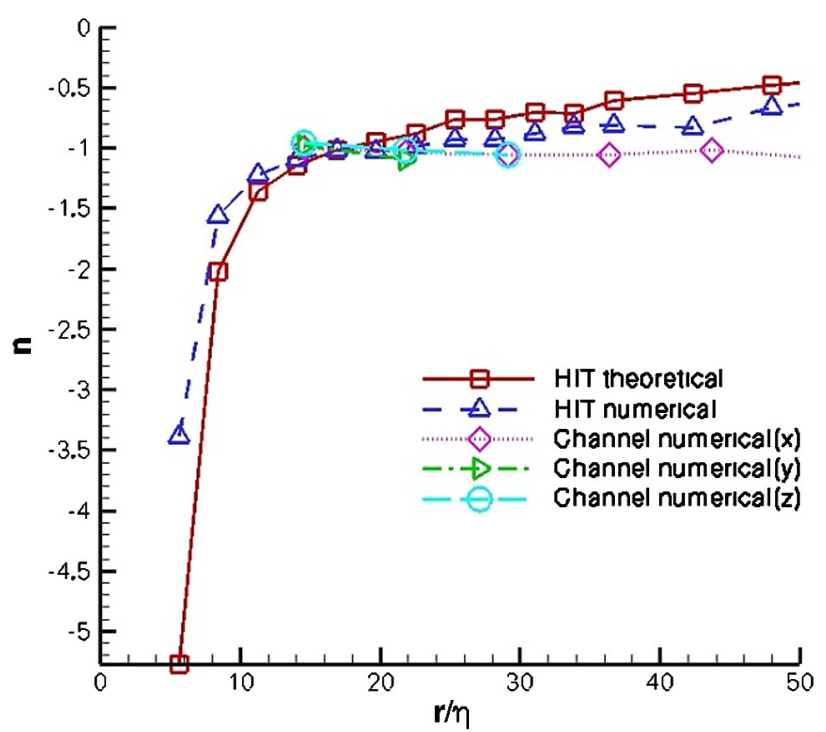

(b)

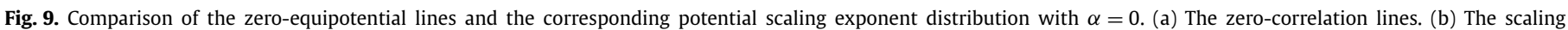
exponents.

law of turbulent flows regardless of the large-scale anisotropic effects. We remark that the different behavior in the streamwise direction might be a result of the normalization of length scales. We calculate the Kolmogorov scale in each $x-z$ plane by using a spherical average of all directions, while this may be not appropriate since in the streamwise direction there may be larger structures. This problem is beyond the scope of the current letter and will be investigated in our future work. Indeed, the problem of calculating the Kolmogorov scale in anisotropic turbulence does not affect the present analysis: instead of the values of the length scales, as stated above, we are more interested in the scaling of the zero-correlation lines, which are not affected by the normalization problem. From Fig. 9(b), if we focus on the region where $r / \eta \sim 20$, which is approximately the starting of the inertial range [20], the scaling exponent values in any direction $(x, y$ or $z)$ fluctuate around -1.0 , which is in agreement with the HIT results. Differences in other regions could be explained as the effect of energy-containing structures. In LES we are more interested in the scaling at the transition between dissipative and inertial ranges [18], therefore an agreement around $r / \eta \sim 20$ might be interesting. It means that when the three-point velocity increment correlation is zero, the parameter $r$ is always approximately inversely proportional to $l$, i.e., $r \propto l^{-1}$. This law is, at least in current test cases, not affected by the large-scale anisotropic structures.

\section{Discussion and conclusion}

Aiming at providing modeling tools for LES, researchers have been searching for multiscale correlations in complex flows, for instance in Ref. [13]. However, we would like to ask that in these multiscale correlations, which part is a consequence of the wall effect and which part is the endogenetic one that exists in all types of turbulent flows including the HIT. The present letter is therefore an attempt to clarify the endogenetic multiscale correlation by 
investigating the three-point velocity increment correlation function. Two types of correlation, i.e., the inertial-inertial type and the inertial-dissipative type, are clarified to be both endogenetic in HIT. These two types lead to negative and positive values of the three-point correlation, respectively. Therefore, there exists a zerocorrelation point, which corresponds to the equilibrium between these two types of correlation and should be helpful in the studies of LES. For example, if we revisit the work of Marusic et al. [13] in these zero-correlation points, the endogenetic correlation can be excluded. In addition, we may also employ the zero-correlation in subgrid modeling, in order to allow a respective modeling of the inertial-inertial and inertial-dissipative interactions.

Therefore, the contributions of the current letter can be concluded as follows.

(1) Our work expands the results of Benzi et al. [15] from one dimension to three dimensions. In HIT the theoretical equations are derived from the tensor analysis and the classical theory of second-order structure functions. The results are compared with DNS results and are in good agreement.

(2) Considering LES, where the smallest resolvable scale is in the inertial range, two types of three-point correlation are summarized, i.e., the inertial-inertial type and the inertial-dissipative type. They are both endogenetic in turbulent flows, yet they have different three-point multiscale behaviors: the values of three-point correlation are of different signs.

(3) The zero-correlation point is then introduced to represent the multiscale equilibrium. This zero-correlation point can only exist when the angle of the three points $\alpha<\pi / 2$. In the parameter space, DNS results of HIT and channel Couette flow show that, when $\alpha=0$ and $r$ or $l$ is in inertial range, the scalings of the zero-correlation lines are similar. This might imply the universality of this zero-correlation scaling.

(4) This work could be expected to be a dependable theoretical base for creating multi-scales subgrid models in LES, as discussed in the above parts. Future researches in the domain of passive scalar turbulence [24] might be also interesting.

In the end, we would like to clarify the difference between this three-point correlation and the classical triad interaction in spectral space [25]. Although the triangles are similar, the physical backgrounds of them are different. The triad interaction is a description of energy transfer, corresponding to a third-order velocity variable. By contrast, the three-point correlation is a second-order function, which is related to the energy of turbulence.

\section{Acknowledgements}

This work is supported by the National Natural Science Foundation in China (11202013, 51136003), the National Basic Research Program of China (2012CB720200) and the open project of the state key laboratory of nonlinear mechanics (LNM).

\section{References}

[1] C. Meneveau, Annu. Rev. Fluid Mech. 32 (2000) 101146.

[2] L. Fang, PhD thesis, Ecole Centrale de Lyon, 2009.

[3] G.X. Cui, H.B. Zhou, Z.S. Zhang, L. Shao, Phys. Fluids 16 (2004) 2835.

[4] L. Shao, Z.S. Zhang, G.X. Cui, C.X. Xu, Phys. Fluids 17 (2005) 115106.

[5] G.X. Cui, C.X. Xu, L. Fang, L. Shao, Z.S. Zhang, J. Fluid Mech. 582 (2007) 377.

[6] A.N. Kolmogorov, Proc.: Math. Phys. Sci. 30 (1941) 15.

[7] A.N. Kolmogorov, J. Fluid Mech. 13 (1962) 82.

[8] Z.S. She, E. Leveque, Phys. Rev. Lett. 72 (1994) 336.

[9] R.A. Antonia, J. Kim, L.W. Browne, J. Fluid Mech. 233 (1991) 369.

[10] S. Kurien, K.R. Sreenivasan, Phys. Rev. E 62 (2000) 2206.

[11] C.M. Casciola, P. Gualtieri, B. Jacob, R. Piva, Phys. Fluids 19 (2007) 101704.

[12] Y. Li, J. Turbul. 12 (2011).

[13] I. Marusic, R. Mathis, N. Hutchins, Science 329 (2010) 5988.

[14] V.S. L'vov, I. Procaccia, Phys. Rev. Lett. 77 (1996) 101103.

[15] R. Benzi, L. Biferale, F. Toschi, Phys. Rev. Lett. 80 (1998) 3244.

[16] G.K. Batchelor, Math. Proc. Camb. Philos. Soc. 47 (1951) 359.

[17] D. Lohse, A. Muller-Groeling, Phys. Rev. Lett. 74 (1995) 1747.

[18] C. Meneveau, Phys. Rev. E 54 (1996) 3657.

[19] L. Chevillard, B. Castaing, E. Lévêque, A. Arneodo, Physica D 218 (2006) 77.

[20] L. Fang, W.J.T. Bos, X.Z. Zhou, L. Shao, J.P. Bertoglio, Acta Mech. Sin. 26 (2010) 151.

[21] J.O. Hinze, Turbulence, McGraw-Hill Series in Mechanical Engineering, McGraw-Hill, Cambridge, 1975.

[22] T. Watanabe, T. Gotoh, New J. Phys. 6 (2004) 40.

[23] L. Fang, L. Shao, J.P. Bertoglio, L.P. Lu, Z.S. Zhang, J. Turbul. 12 (2011) 1.

[24] L. Mydlarski, A. Pumir, B.I. Shraiman, E.D. Siggia, Z. Warhaft, Phys. Rev. Lett. 81 (1998) 4373.

[25] R.H. Kraichnan, Phys. Fluids 10 (1967) 1417. 